\title{
Study of Mobile Oxygen in Ordered/Disordered \\ Nonstoichiometric Sr-Gd-Cobaltate \\ by Simultaneous Thermal Analysis
}

\author{
Sergei N. Vereshchagin*a, \\ Vyacheslav A. Dudnikov ${ }^{\mathrm{b}}$ and Leonid A. Solovyov ${ }^{\mathrm{a}}$ \\ ${ }^{a}$ Institute of Chemistry and Chemical Technology SB RAS \\ FRC "Krasnoyarsk Science Center SB RAS" \\ 50/24 Akademgorodok, Krasnoyarsk, 660036, Russia \\ ${ }^{b}$ Kirensky Institute of Physics SB RAS \\ 50/38 Akademgorodok, Krasnoyarsk, 660036, Russia
}

Received 20.03.2017, received in revised form 14.06.2017, accepted 03.09.2017

\begin{abstract}
Simultaneous thermal analysis (DSC/TG/mass-spectroscopy) under variable pressure was applied to study properties of mobile oxygen in cubic disordered and tetragonal A-site ordered $S r$-Gd-cobaltate $(\mathrm{Sr} / \mathrm{Gd}=4)$. It was shown that A-site cation ordering results in a decrease of an enthalpy of oxygen elimination, predominant localization of created anion vacancies only at one of four unequivalent crystallographic $O$-sites and depletion of amount of mobile oxygen, which can be removed at a reduced pressure. The observed properties of mobile oxygen and catalytic activity in reaction of methane deep oxidation for ordered/disordered perovskite phases are discussed in connection with a local environment of $\mathrm{O}^{2-}$ crystallographic sites with a different number of $\mathrm{Gd}^{3+}$ cations nearby.
\end{abstract}

Keywords: disordered perovskite, cobaltate, DSC, TG, crystal structure, mobile oxygen.

DOI: $10.17516 / 1998-2836-0031$.

(C) Siberian Federal University. All rights reserved

* Corresponding author E-mail address: snv@icct.ru 


\title{
Изучение подвижного кислорода
}

\section{в упорядоченном/разупорядоченном нестехиометрическом кобальтате $\mathrm{Sr}-\mathrm{Gd}$ методом синхронного термического анализа}

\author{
С.Н. Верещагин ${ }^{\mathrm{a}}$, В.А. Дудников ${ }^{\boldsymbol{\sigma}}$, Л.А. Соловьев ${ }^{\mathrm{a}}$ \\ ${ }^{a}$ Институт химии и химической технологии СО РАН \\ ФИЦ «Красноярский научный центр СО РАН» \\ Россия, 660036, Красноярск, Академгородок, 50/24 \\ ${ }^{6}$ Институт физики им. Л.В. Киренского СО РАН \\ Россия, 660036, Красноярск, Академгородок, 50/38
}

Методом синхронного термического анализа (ДСК/ТГ/масс-спектрометрия) при переменном парииальном давлении кислорода изучены разупорядоченная кубическая и упорядоченная по A-позициям тетрагональная фазы $S r$ - $G d$-кобальтата ( $S r / G d=4)$. Показано, что упорядочение $A$-катионов приводит к снижению энтальпии отщепления $O_{2}$, преимущественной локализации образовавшейся вакансии в одной из четырёх неэквивалентных позищий аниона $O^{2-}$ и снижению количества подвижного кислорода, удаляемого при изменении парииального давления $O_{2}$. Наблюдаемые различия в свойствах мобильного кислорода и каталитической активности в реакции глубокого окисления метана для упорядоченной/разупорядоченной фазы перовскита обсуждены с позищий изменения доли анионов $O^{2-}$ с различным локальным окружением, содержащим от 0 до 4 катионов гадолиния.

Ключевые слова: разупорядоченный перовскит, кобальтат, ДСК, ТГ, кристаллическая структура, подвижный кислород.

\section{Введение}

Замещенные редкоземельные кобальтаты $M_{x} \operatorname{Ln}_{1-x} \mathrm{CoO}_{3-\delta}(M-$ щелочноземельный металл, $L n$ - лантаноид) со структурой перовскита - перспективные системы для применения в каталитических реакциях глубокого и парциального окисления углеводородов [1]. Общепринято, что энергия связи кислорода играет определяющую роль в окислительном катализе, а определение факторов, влияющих на характер и энергию связи поверхностного кислорода, является важным этапом целенаправленного синтеза активных катализаторов окислительных превращений углеводородов и регулирования их селективности. Для рассматриваемых перовскитных систем $M_{x} L n_{1-x} \mathrm{CoO}_{3-\delta}$ свойства поверхностного кислорода и его подвижность в решетке определяются рядом факторов, таких как химическая природа катионов в А и В позициях структуры перовскита $\mathrm{ABO}_{3}$, наличие и количество кислородных вакансий [2], легкость удаления кислорода с поверхности или из решетки [3], структура электронных уровней вблизи уровня Ферми, заселенность и электронная конфигурация d-уровня переходного В-металла [4], степень ковалентности связи переходного В-металла 
с кислородом [5], а также особенностями микродоменной структуры в приповерхностных слоях перовскитов [6].

Недавно нами было показано [7, 8], что на уровень каталитической активности соединений $\mathrm{Sr}_{\mathrm{x}} \mathrm{Gd}_{1-\mathrm{x}} \mathrm{CoO}_{3-\delta}$ существенное влияние оказывает характер распределения каталитически “неактивных" катионов $\mathrm{Sr} / \mathrm{Gd}$ в А-позициях перовскитной структуры. На основании литературных данных [9] было предположено, что существенное увеличение каталитической активности разупорядоченного по А-позициям перовскита по сравнению с образцом с упорядоченным распределением А-катинов связано с изменением подвижности кислорода в решетке. Однако до настоящего времени в литературе не опубликованы экспериментальные исследования, посвященные влиянию упорядочения катионов в А-позиции на характеристики подвижного кислорода в Sr-Gd-кобальтатах. Традиционно одним из наиболее мощных методов получения полной информации о поверхностных кислородных формах и кинетических свойствах поверхностного и объемного кислорода является изотопный обмен с использованием ${ }^{18} \mathrm{O}_{2}$, однако этот метод трудоемкий и дорогостоящий. Высокая мобильность кислорода, присутствующего в исследуемой системе, позволяет надеяться на то, что его свойства могут быть исследованы прямыми методами термического анализа с получением информации об энтальпии процессов и количествах подвижного кислорода.

Цель настоящей работы - адаптация метода синхронного термического анализа для получения количественных характеристик подвижного кислорода на примере хорошо окристаллизованных образцов $\mathrm{Sr}_{0.8} \mathrm{Gd}_{0.2} \mathrm{CoO}_{3-\delta}$ с упорядоченным/разупорядоченным распределением $\mathrm{Sr} / \mathrm{Gd}$, а также анализ структурных особенностей этих перовскитов для выявления возможных причин различий в каталитической активности.

\section{Экспериментальная часть}

Поликристаллический образец $\mathrm{Sr}_{0.8} \mathrm{Gd}_{0.2} \mathrm{CoO}_{3-\delta}$ был получен по стандартной керамической технологии на воздухе при температуре $1473 \mathrm{~K}$ из оксидов $\mathrm{Gd}_{2} \mathrm{O}_{3}, \mathrm{Co}_{3} \mathrm{O}_{4}$ (чистота 99.9 \%) и карбоната стронция $\mathrm{SrCO}_{3}$ (чистота $99 \%$ ), детали синтеза подробно описаны ранее [10]. Образец с упорядоченным распределением катионов $\mathrm{Sr} / \mathrm{Gd}(\mathrm{y}-\mathrm{S} 8 \mathrm{G} 2)$ получен медленным охлаждением $\left(2{ }^{\circ}\right.$ мин $\left.^{-1}\right)$ на воздухе таблеток $\mathrm{Sr}_{0.8} \mathrm{Gd}_{0.2} \mathrm{CoO}_{3-\delta}$ от $1473 \mathrm{~K}$ до комнатной температуры; образец с разупорядоченным распределением катионов (p-S8G2) - закалкой от $1473 \mathrm{~K}\left(\sim 100{ }^{\circ} \mathrm{c}^{-1}\right)$. Для стабилизации содержания кислорода образцы дополнительно выдерживались 6 ч на воздухе при 773 К.

Синхронный термический анализ проводился на приборе Netzsch STA Jupiter 449C с масcспектральным анализатором Aeolos QMS403C в потоке газовой смеси $\mathrm{O}_{2}-\mathrm{Ar}\left(100 \mathrm{~cm}^{3} \mathrm{MnH}^{-1}\right)$, общее давление в ДСК/ТГ ячейке составляло около 100 кПа. Измерения проводились в платиновых тиглях, для анализа использовалась фракция 0.1-0.16 мм; масса образца 27.00 мг. Коэффициент чувствительности сенсора определялся из теплоемкости стандартного сапфирового диска по методу [11], ошибка определения $\Delta$ Н составляла $4 \%$.

Содержание кислорода в исходных перовскитах $\mathrm{Sr}_{0.8} \mathrm{Gd}_{0.2} \mathrm{CoO}_{3-\delta}$ определено из убыли массы образца при его восстановлении водородом по методике [12], погрешность определения нестехиометрии $\delta$ составляла \pm 0.01 . Вариации $\delta$ в экспериментах со сменой составов смесей рассчитаны на основании изменения массы образца. 
Рентгенофазовый и рентгеноструктурный анализы $\mathrm{y}-\mathrm{S} 8 \mathrm{G} 2$ и $\mathrm{p}-\mathrm{S} 8 \mathrm{G} 2$ перовскитов проводили с использованием дифрактометра PANalytical X'Pert PRO $(\mathrm{CoK} \alpha)$ в интервале углов $2 \theta 10-140^{\circ}$. Обработка результатов проведена с применением полнопрофильного анализа поликристаллических веществ по методу Ритвельда и методу минимизации производной разности [13].

Удельная поверхность образцов определялась по многоточечному методу БЭТ из данных по низкотемпературной сорбции азота.

Каталитические свойства при превращении метана в присутствии кислорода определялись в микрокаталитической проточной установке BiCatFlow в кварцевом микрореакторе со стационарным слоем катализатора (фракция 0.1-0.2 мм, 100 мг) при атмосферном давлении с использованием хроматографического анализа продуктов (многоколоночный хроматограф Agilent). Состав реакционной смеси (здесь и далее об. \%) $\mathrm{CH}_{4}: \mathrm{O}_{2}: \mathrm{He}=27: 13: 60$.

\section{Результаты и обсуждение}

Как было продемонстрировано ранее [14], при нагревании нестехиометрического двойного перовскита $\mathrm{Sr}_{0.8} \mathrm{Gd}_{0.2} \mathrm{CoO}_{3-\delta}$ в потоке $20 \% \mathrm{O}_{2}$-Ar при температурах выше $773 \mathrm{~K}$ наблюдалось заметное выделение кислорода из образца вследствие протекания реакции (1), которая в обозначениях Крёгера и Винка для кобальтатов может быть записана как образование анионной (кислородной) вакансии V••

$$
\begin{aligned}
& \mathrm{Sr}_{0.8} \mathrm{Gd}_{0.2} \mathrm{CoO}_{3-\delta 1}=\mathrm{Sr}_{0.8} \mathrm{Gd}_{0.2} \mathrm{CoO}_{3-\delta 2}+\left(\delta_{2}-\delta_{1}\right) / 2 \mathrm{O}_{2}+\Delta \mathrm{H}, \\
& \mathrm{O}_{\mathrm{O}}^{\mathrm{x}}+2 \mathrm{Co}_{\mathrm{Co}}=1 / 2 \mathrm{O}_{2}+\mathrm{V}^{\bullet \bullet}{ }_{\mathrm{O}}+2 \mathrm{Co}^{\mathrm{x}}{ }_{\mathrm{Co}},
\end{aligned}
$$

где обозначения $\mathrm{Co}_{\text {Сo }}, \mathrm{Co}_{\mathrm{Co}}^{\mathrm{x}}$ и $\mathrm{O}_{\mathrm{O}}^{\mathrm{x}}$ соответствуют ионам $\mathrm{Co}^{4+}, \mathrm{Co}^{3+}$ и $\mathrm{O}^{2-}$ на позициях кобальта и кислорода кристаллической структуры соответственно.

К аналогичным результатам приводило также снижение парциального давления кислорода в смеси при постоянной температуре. Для изучения возможности использования ТГ/ДСКметода для характеристики подвижного кислорода в перовскитной структуре были проведены эксперименты по ступенчатому изменению парциального давления $\mathrm{P}_{\mathrm{O} 2}$ в газовой фазе. На рис. 1 приведен типичный профиль концентрации кислорода в ТГ/ДСК ячейке при ступенчатом изменении $\mathrm{P}_{\mathrm{O} 2}$ и происходящие при этом изменения массы (ТГ) и теплового потока (ДСК). Как следует из данных рис. $1 a$ при замене смеси $13 \% \mathrm{O}_{2}$-Ar на аргон с остаточным содержанием кислорода 0.01 \% наблюдалось быстрое снижение массы образца вследствие протекания эндотермической реакции (1), причем постепенное выделение кислорода происходило и после стабилизации $\mathrm{P}_{\mathrm{O} 2}$ в течение 20 мин. Суммарное изменение массы составило $-0.46 \%$, что соответствует конечной величине нестехиометрии $\delta_{2}=0.35(\Delta \delta=0.06)$.

Обратное переключение смеси с $0.01 \% \mathrm{O}_{2}$-Ar на $13 \% \mathrm{O}_{2}$-Ar приводило к резкому эндотермическому набору массы до исходного значения (рис. 1a). Форма пиков ДСК кривых восстановления и реокисления образца существенно различаются, что указывает на более быстрое протекание процесса поглощения $\mathrm{O}_{2}$ по сравнению с его удалением в используемых условиях. Очевидно, что данное проведение процесса $\left(13 \% \mathrm{O}_{2}=>0.01 \% \mathrm{O}_{2}\right)$ затруднительно использовать для количественной характеристики подвижного кислорода, поскольку в течение длительно-

$$
-349-
$$




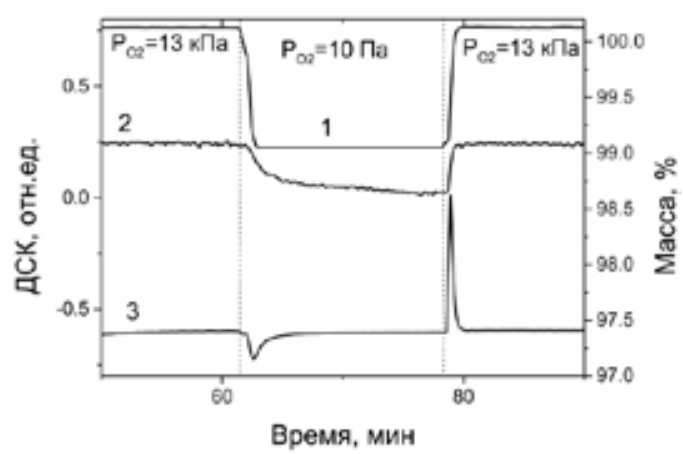

a

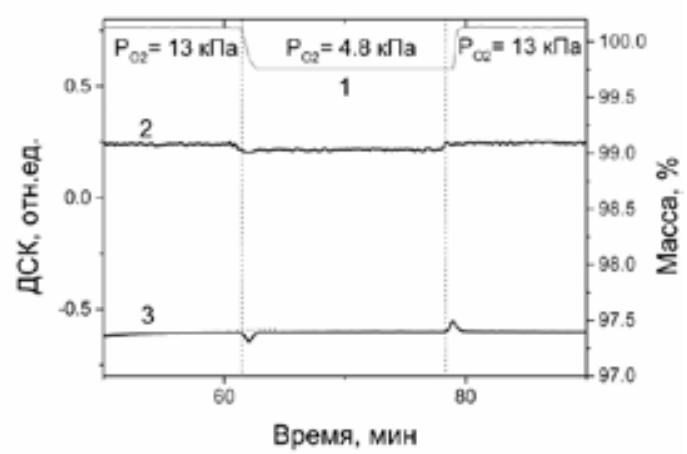

б

Рис. 1. Профили парциального давления кислорода (1), изменение ТГ (2) и ДСК (3) сигналов при ступенчатом изменении состава газовой смеси, моменты переключения обозначены вертикальными линиями. Образец р-S8G2, $773 \mathrm{~K}$

Fig. 1. Partial pressure of oxygen (1), TG (2) and DSC (3) signals under abrupt change of mixture composition. Vertical lines designate time of switching. p-S8G2, $773 \mathrm{~K}$

го времени наблюдается постоянный дрейф массы, не позволяющий строго определить как количество удаленного кислорода, так и энтальпию процесса. Кроме того, вследствие значительного изменения нестехиометрии следует принимать во внимание возможное изменение энтальпии процесса (1) со снижением величины $\delta$.

На рис. 16 приведены результаты эксперимента по ступенчатому изменению состава смесей в последовательности $13 \% \mathrm{O}_{2}-\mathrm{Ar}=>4.8 \% \mathrm{O}_{2}-\mathrm{Ar}=>13 \% \mathrm{O}_{2}$ - $\mathrm{Ar}$. В этом случае после снижения концентрации $\mathrm{O}_{2}$ наблюдалось быстрая стабилизация массы, а ДСК пики окислениявосстановления имели близкую форму. Однако общие изменения массы и энтальпии были невелики $(\Delta \mathrm{m}=0.048 \%, \Delta \delta=0.01)$ и находились вблизи предела чувствительности используемой аппаратуры ТГ/ДСК. Большая ошибка определения $\Delta \mathrm{m}$ и $\delta$ приведет к значительной неопределенности в расчетных значениях удельной энтальпии отщепления кислорода (реакции (1)), особенно в случае образцов с более низким содержанием подвижного кислорода по сравнению p-S8G2 (в частности, y-S8G2).

Технические характеристики используемой аппаратуры термического анализа (Netzsch STA Jupiter 449C в комплекте с приставкой дозировки потоков PulseTA) не давали возможности варьировать концентрации смесей в широком интервале, поэтому была применена методика с “импульсной” дозировкой Ar. Для этогов измерительной ячейке поток смеси $13 \% \mathrm{O}_{2}$-Ar на 1 мин. заменялся эквивалентным этого в потокомаргона. В связи со значительным объемом измерительной части и подводящих газовых потоком аргона коммуникаций входной и выходной фронты аргона уширялись, а результирующий профиль концентрации кислорода был близок по форме к гауссовой кривой (рис. 2, кривая 1 ); минимальное значение содержания $\mathrm{O}_{2}$ (определенное по масс-спектральному анализу отходящих газов) составляло около $1.3 \%$.

Симбатно изменению содержания кислорода наблюдалось снижение/возрастание массы образца и вариация теплового потока (рис. 2, кривые 2-5), причем соответствующие изменения происходили практически одновременно с $\mathrm{P}_{\mathrm{O} 2}$. Величины $\Delta \mathrm{m}$ и площади под кривой ДСК четко 


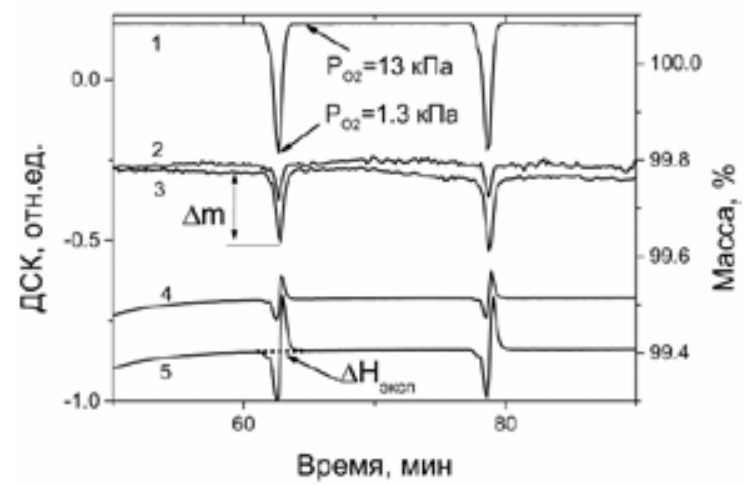

Рис. 2. Профиль концентрации кислорода $(1)$, ТГ $(2,3)$ и ДСК $(4,5)$ сигналы при импульсном изменении состава газовой смеси (ТГ- и ДСК-кривые сдвинуты для удобства восприятия). 2,4 - y-S8G2; 3,5 p-S8G2

Fig. 2. Partial pressure of oxygen (1), TG $(2,3)$ and DSC $(4,5)$ signals under "pulse" change of mixture composition. TG and DSC curves are shifted for convenience. 2,4 - y-S8G2; 3,5 - p-S8G2

фиксировались, обеспечивая относительную ошибку воспроизводимости менее 4 \% для наименьшего из полученных пиков.

Описанный “импульсный” подход был использован для определения удельной энтальпии удаления $\mathrm{O}_{2}$ и сопоставления относительного количества подвижного кислорода для упорядоченной и разупорядоченной структур (рис. 2).

В качестве характеристик подвижного кислорода рассчитывались значения:

$\Delta \mathrm{n}$ - рассчитанное на основании $\Delta \mathrm{m}$ (рис. 2) количество ( $\mu г$-атом $\left.\mathrm{O} \Gamma^{-1}\right)$ кислорода, удаляемого в “импульсном" эксперименте;

$\mathrm{N}_{\mathrm{m}}$ - число удаляемых монослоев кислорода в предположении, что емкость монослоя составляет $0.225 \cdot 10^{-5} \Gamma$-атом $\mathrm{O} \Gamma^{-1}$, при величине удельной поверхности $\mathrm{S}_{\mathrm{yд}}=0.1 \mathrm{~m}^{2} \Gamma^{-1}$;

$\Delta \mathrm{H}=\Delta \mathrm{H}_{\text {экп }} / \Delta \mathrm{n}-$ энтальпия отщепления кислорода (кДж г-атом ${ }^{-1} \mathrm{O}$ ).

На рис. 2 приведены экспериментальные ТГ- и ДСК-кривые для образцов p-S8G2 и p-S8G2, а в табл. 1 - рассчитанные величины $\Delta \mathrm{n}, \mathrm{N}_{\mathrm{m}}$ и $\Delta \mathrm{H}$. Дополнительно приведены также значения константы скорости $k_{773}$ реакции полного окисления метана (2), изученной ранее [7].

$$
\mathrm{CH}_{4}+2 \mathrm{O}_{2}=>\mathrm{CO}_{2}+2 \mathrm{H}_{2} \mathrm{O}
$$

Полученные значения $\Delta \mathrm{H}$ находятся в диапазоне, отмеченном в работе [15] для расчетной энтальпии реакции отщепления кислорода из соединения $\mathrm{PrBaCo}_{2} \mathrm{O}_{6-\delta}$. Однако в отличие от них приведенные в табл. 1 значения определены экспериментально, не требуют знания энтальпии образования исходных соединений и свободны от допущений о неизменности теплоемкости с вариацией $\delta$.

Из данных табл. 1, следует, что наблюдаются заметные отличия упорядоченного и разупорядоченного перовскитов как по количеству подвижного кислорода, так и по энтальпии его удаления. Хотя в ходе экспериментов вариация нестехиометрии $\delta$ была невелика, изменения затрагивали значительную часть твердого тела (44 и 19 монослоев для p- и y-S8G2 соответственно), что соответствует очень высокой подвижности кислорода. Эти данные подтверж- 
Таблица 1. Нестехиометрия $(\delta)$, параметры структуры перовскита $\mathrm{Sr}_{0.8} \mathrm{Gd}_{0.2} \mathrm{CoO}_{3-\delta}$ в различных состояниях, количество и свойства подвижного кислорода

Table 1. Non-stoichiometry $(\delta)$, crystal structure parameters of ordered/disordered $\mathrm{Sr}_{0.8} \mathrm{Gd}_{0.2} \mathrm{CoO}_{3-\delta}$, amount and properties of mobile oxygen

\begin{tabular}{|c|c|c|}
\hline & Разупорядоченный & Упорядоченный \\
\hline Код образца & p-S8G2 & $\mathrm{y}-\mathrm{S} 8 \mathrm{G} 2$ \\
\hline$\delta^{*}$ & 0.29 & 0.37 \\
\hline $\begin{array}{l}\text { Структура } \\
\text { (при } 298 \text { К) }\end{array}$ & $\begin{array}{l}\text { Кубическая } \\
\text { Pm } 3 m\end{array}$ & $\begin{array}{c}\text { Тетрагональная } \\
\text { I4/mmm }\end{array}$ \\
\hline $\mathrm{a}, \AA$ & $3.8342(1)$ & $7.6920(26)$ \\
\hline $\mathrm{c}, \AA$ & & $15.321(8)$ \\
\hline$\Delta \mathrm{m}, \%(\Delta \delta)^{* *}$ & $0.148(0.03)$ & $0.073(0.01)$ \\
\hline$\Delta \mathrm{n}, \mu \Gamma$-атом $\mathrm{O} \Gamma^{-1}$ & 98.8 & 43.8 \\
\hline $\mathrm{N}_{\mathrm{m}}$ (монослой) & 44 & 19 \\
\hline$\Delta \mathrm{H}$, кДж г-атом ${ }^{-1} \mathrm{O}$ & 46.5 & 43.3 \\
\hline$k_{773} \cdot 10^{8}$,моль $\Gamma^{-1} \mathrm{c}^{-1}$ атм $^{-1, * * *}$ & 39.3 & 6.4 \\
\hline
\end{tabular}

* - после выдержки на воздухе при 773 К и охлаждении до 298 К (по данным ТГ);

** - изменение массы (нестехиометрии) в “импульсном эксперименте”;

*** - константы скорости реакции (2) при 773 К.

дают ранее высказанное предположение $[7,8]$, что более высокая каталитическая активность p-S8G2 (39.3·10-8 против 6.4.10-8 моль $\Gamma^{-1} \mathrm{c}^{-1} \mathrm{aTM}^{-1}$ для $\mathrm{y}-\mathrm{S} 8 \mathrm{G} 2$, табл. 1) обусловлена большим количеством подвижного кислорода. Примечательно, что величина $\Delta \mathrm{H}$ для p-S8G2 превосходит соответствующее значение для $\mathrm{y}-\mathrm{S} 8 \mathrm{G} 2$, что может свидетельствовать о различном характере центров, из которых удаляется кислород, и отличиях в энергетике стабилизации возникающих вакансий в у- и р-S8G2.

Для выяснения возможных причин влияния распределения катионов в А-позиции $\mathrm{Sr}_{0.8} \mathrm{Gd}_{0.2} \mathrm{CoO}_{3-\delta}$ на подвижность кислорода в объеме перовскита были проанализированы структуры упорядоченной и разупорядоченной фазы с точки зрения отличий в локальном окружении анионов $\mathrm{O}^{2-}$.

На рис. 3 показано основанное на результатах рентгеноструктурного анализа расположение катионов в разупорядоченной кубической и упорядоченной тетрагональной фазах. В кубическом p-S8G2 все А-позиции равноценны и заняты статистически распределенными катионами $\mathrm{Sr}^{2+} / \mathrm{Gd}^{3+}$, анионы $\mathrm{O}^{2-}$ и анионные вакансии равномерно заполняют эквивалентные позиции в вершинах октаэдров (рис. $3 a$ ). Структура тетрагонального y-S8G2 аналогична структуре двойных кобальтатов стронция с редкоземельными металлами [16], в которой А-катионы частично упорядочены в “колоннах" [17]. В этой структуре имеются три неэквивалентных позиции А-катиона, две из которых заняты ионами $\mathrm{Sr}^{2+}$; третья позиция заполняется случайно выбранными катионами $\mathrm{Sr}^{2+}$ или $\mathrm{Gd}^{3+}$ (рис. 36). В структуре присутствуют четыре неэквивалентные позиции кислорода (рис. 36, позиции О1-О4), причем в соответствии результатами наших исследований при 298 К анионные вакансии в образце y-S8G2 локализованы исключительно в позиции O2, что согласуется с литературными данными [16]; заполнение соответствующей позиции, полученное при обработке порошковых 


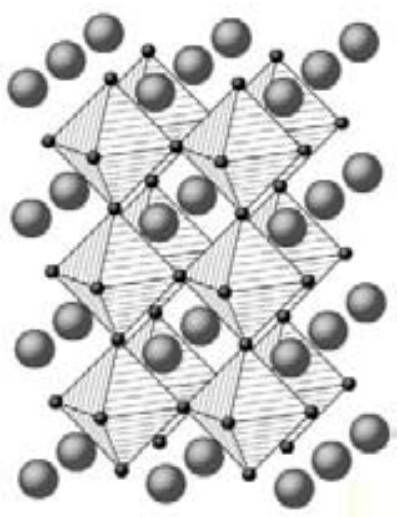

A

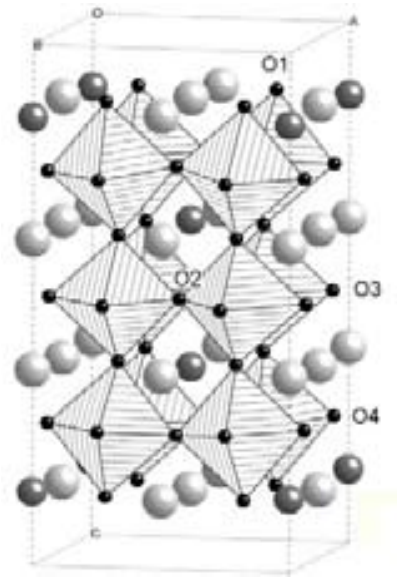

B

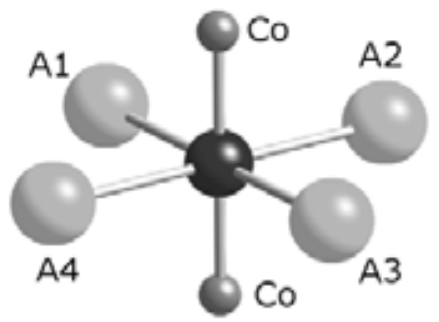

Рис. 3. Изображение кубической (А) и тетрагональной (В) структуры $\mathrm{Sr}_{0.8} \mathrm{Gd}_{0.2} \mathrm{CoO}_{3-\delta}$. Октаэдры соответствуют катионам $\mathrm{Co}^{\mathrm{n}+}$, чёрные сферы соответствуют положению анионов $\mathrm{O}^{2-} /$ анионных вакансий, светло-серые - позициям $\mathrm{Sr}^{2+}$, темно-серые - позициям катионов метками O1-O4 обозначены неэквивалентные позиции кислорода. (C) - локальное окружение аниона кислорода в структуре перовскита (A1-A4 - катионы $\mathrm{Sr}^{2+} / \mathrm{Gd}^{3+}$ )

Fig. 3. Cubic (A) and tetragonal (B) structure of $\mathrm{Sr}_{0.8} \mathrm{Gd}_{0.2} \mathrm{CoO}_{3-\delta}$. Octahedra are $\mathrm{Co}^{\mathrm{n}+}$ cations, black spheres $\mathrm{O}^{2-/ a n i o n ~ v a c a n c i e s, ~ l i g h t-g r a y ~ s p h e r e s ~ a r e ~} \mathrm{Sr}^{2+}$, dark-gray spheres are $\mathrm{Sr}^{2+} / \mathrm{Gd}^{3+}$, O1-O4 are nonequivalent oxygen sites. (C) - local environment of perovskite oxygen, A1-A4 are $\mathrm{Sr}^{2+} / \mathrm{Gd}^{3+}$ cations

рентгенограмм по методу Ритвельда, составило 0.23. С учетом мультиплетности позиций кислорода для $\mathrm{y}-\mathrm{S} 8 \mathrm{G} 2$ это дает значение нестехиометрии $\delta=0.385$, которое удовлетворительно согласуется с величиной $\delta=0.37$, определенной методом ТГ при восстановлении водородом.

Для обоих типов рассматриваемых структур анионы $\mathrm{O}^{2-}$ окружены шестью катионами, образующими в общем случае искаженное октаэдрическое окружение: 2 катиона $\mathrm{Co}^{\mathrm{nt}}$ на полюсах и четыре А-катиона A1-A4 $\left(\mathrm{Sr}^{2+} / \mathrm{Gd}^{3+}\right)$ в экваториальной плоскости (рис. $3 c$ ).

В разупорядоченном кубическом перовските все позиции кислорода кристаллографически эквивалентны, однако локальные окружения конкретных анионов $\mathrm{O}^{2-}$ будут отличаться. Это следует из того, что позиции A1-А4 занимают случайным образом выбранные катионы $\mathrm{Sr}^{2+}$ или $\mathrm{Gd}^{3+}$, формируя все возможные наборы экваториальных катионов, от четырёх $\mathrm{Sr}^{2+}$ до четырёх $\mathrm{Gd}^{3+}$, включая промежуточные варианты с одним, двумя и тремя катионами $\mathrm{Gd}^{3+}$. Для используемой модели (рис. 3c) и в предположении о статистическом распределении $\mathrm{Sr} / \mathrm{Gd}$ вероятность $p(x)$ образования центра, содержащего $x$ атомов катиона A (например, $\mathrm{Gd}^{3+}$ ) выражается формулой (3)

$$
p(x)=\left(\frac{n}{x}\right) \alpha^{x}(1-\alpha)^{n-x} ;\left(\frac{n}{x}\right)=\frac{n !}{x !(n-x) !},
$$

где $n$ - число позиций; $\alpha$ - доля катиона А.

Более сложная ситуация реализуется для упорядоченного перовскита, поскольку в нем присутствуют четыре неэквивалентных позиции кислорода, окружение которых (A1-A4) в пер- 
Таблица 2. Локальное окружение анионов кислорода в кубической и тетрагональной структуре (рис. 3)

Table 2. Local environment of oxygen anions in cubic and tetragonal structures. (Fig.3)

\begin{tabular}{|c|c|c|c|c|c|c|}
\hline \multirow{2}{*}{$\begin{array}{l}\text { Позиция О }{ }^{2-} \\
\text { (рис. } 3 \mathrm{~A}, \mathrm{~B}) .\end{array}$} & \multirow{2}{*}{$\begin{array}{c}\text { Доля } \\
\text { центров }\end{array}$} & \multirow{2}{*}{$\begin{array}{c}\text { Заполнение } \\
(297 \mathrm{~K})\end{array}$} & \multicolumn{4}{|c|}{ Позиция А-катиона (рис. 3С) } \\
\hline & & & A1 & A2 & A3 & A4 \\
\hline \multicolumn{3}{|c|}{ Разупорядоченный (кубический) } & \multicolumn{4}{|c|}{ Тип А-катиона } \\
\hline $\mathrm{O}$ & 1 & $0.903^{*}$ & $\mathrm{Sr} / \mathrm{Gd}$ & $\mathrm{Sr} / \mathrm{Gd}$ & $\mathrm{Sr} / \mathrm{Gd}$ & $\mathrm{Sr} / \mathrm{Gd}$ \\
\hline \multicolumn{7}{|c|}{ Упорядоченный (тетрагональный) } \\
\hline $\mathrm{O} 1$ & $16 / 48$ & 1 & $\mathrm{Sr} / \mathrm{Gd}$ & $\mathrm{Sr}$ & $\mathrm{Sr}$ & $\mathrm{Sr}$ \\
\hline $\mathrm{O} 2$ & $8 / 48$ & $0.23 * *$ & $\mathrm{Sr} / \mathrm{Gd}$ & $\mathrm{Sr} / \mathrm{Gd}$ & $\mathrm{Sr}$ & $\mathrm{Sr}$ \\
\hline $\mathrm{O} 3$ & $8 / 48$ & 1 & $\mathrm{Sr}$ & $\mathrm{Sr}$ & $\mathrm{Sr}$ & $\mathrm{Sr}$ \\
\hline $\mathrm{O} 4$ & $16 / 48$ & 1 & $\mathrm{Sr} / \mathrm{Gd}$ & $\mathrm{Sr}$ & $\mathrm{Sr}$ & $\mathrm{Sr}$ \\
\hline
\end{tabular}

* - по данным ДСК; ** - по данным РСА.

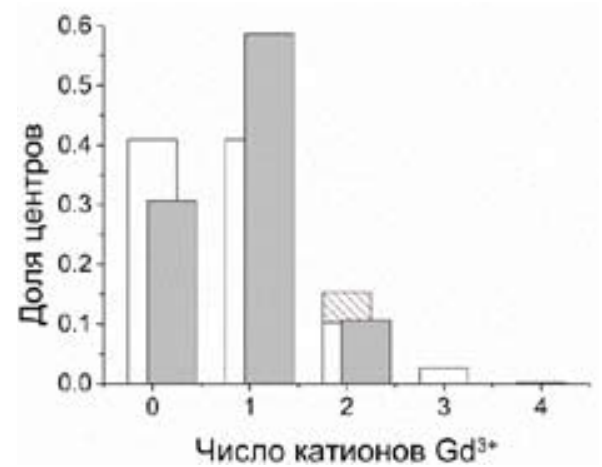

Рис. 4. Расчетная доля позиций кислорода с различным числом катионов $\mathrm{Gd}^{3+}$ для упорядоченного (серые столбцы) и разупорядоченного (белые столбцы) $\mathrm{Sr}_{0.8} \mathrm{Gd}_{0.2} \mathrm{CoO}_{3-\delta}$. Заштрихована часть, соответствующая центрам с двумя атомами гадолиния в транс-конфигурации

Fig. 4. Calculated fraction of oxygen positions with different number of $\mathrm{Gd}^{3+}$ cations for ordered (gray bars) and disordered (white bars) $\mathrm{Sr}_{0.8} \mathrm{Gd}_{0.2} \mathrm{CoO}_{3-\delta}$. A fraction of centers with two $\mathrm{Gd}^{3+}$ in trans-configuration is hatched

вую очередь зависит от положения аниона $\mathrm{O}^{2-}$ в кристаллической структуре и только частично определяется случайным выбором $\mathrm{Sr}$ или Gd (табл. 2).

С использованием формулы (3), мультиплетности центров O1-O4 и структурной информации о катионном окружения позиции кислорода (табл. 2) для упорядоченной и разупорядоченной структур состава $\mathrm{Sr}_{0.8} \mathrm{Gd}_{0.2} \mathrm{CoO}_{3}$ были рассчитаны доли центров $\left(\mathrm{O}^{2-}\right)$ с различным числом катионов $\mathrm{Gd}^{3+}$ (рис. 4). Расчет показал, что наблюдаются существенные отличия в распределении центров различного состава и конфигурации для исследуемых структур, причем trans-конфигурация с двумя атомами Gd (A1A2A3A4 = SrGdSrGd), а также центры с тремя и четырьмя катионами гадолиния присутствуют только в разупорядоченном образце.

Локализация анионной вакансии в упорядоченной структуре при 298 К исключительно в положении О2 (рис. 3, табл. 2) с cis-конфигурацией ближайшего окружения указывает на то, что именно эта позиция является энергетически наиболее выгодной для протекания реакции (1a). Аналогичная сильная зависимость энергии образования вакансий от локального окруже- 
ния продемонстрирована DFT-расчетами на примере $\operatorname{SrTiO}_{3-\delta}(\delta=0.063-0.125)$ при замене одного из четырех катионов $\mathrm{Sr}^{2+}$ на $\mathrm{K}^{+}$или $\mathrm{Na}^{+}[18]$. К сожалению, в данной работе не приводятся данные по энергетике образования вакансий при замене более чем одного атома Sr. B нашем случае повышение температуры и увеличение нестехиометрии $\delta$ приведут, скорее всего, к образованию анионных вакансий также на центрах с другим локальным окружением, однако, как показывают расчеты [19], при этом изменение абсолютной величины энергии образования вакансий в ряду структурно подобных соединений не меняет их качественной относительной стабильности. Поэтому можно предположить, что наблюдаемые отличия в нестехиометрии и свойствах подвижного кислорода на р- и $\mathrm{y}-\mathrm{S} 8 \mathrm{G} 2$ образцах (табл. 1) обусловлены изменением объёмной концентрации анионов $\mathrm{O}^{2-}$, имеющих различный состав и конфигурацию локального окружения А-катионами. Поверхностные координационно-ненасыщенные атомы кислорода, участвующие в каталитической реакции, также с высокой вероятностью будут дифференцированы по соотношению $\mathrm{Sr} / \mathrm{Gd}$ в ближайшем окружении, а концентрация соответствующих поверхностых центров будет пропорциональна их концентрации в объёме, что и обуславливает отличия в каталитической активности р- и $\mathrm{y}-\mathrm{S} 8 \mathrm{G} 2$ образцов. Предложенный подход является, очевидно, очень упрощенным и требует дополнительной проверки на ряде упорядоченных/разупорядоченных систем с другими составами и А-катионами, а для количественного описания полученного эффекта, предсказания физико-химических свойств фаз с различной степенью упорядоченности и определения стабильности анионной вакансии в конкретном окружении необходимо квантово-химическое моделирование с учетом реальной структурной информации о строении локального окружения анионов $\mathrm{O}^{2-}$.

\section{Выводы}

Упорядочение А-катионов в кубическом перовските $\mathrm{Sr}_{0.8} \mathrm{Gd}_{0.2} \mathrm{CoO}_{3-\delta}$ со случайно распределенными катионами $\mathrm{Sr}^{2+} / \mathrm{Gd}^{3+}$ и анионными вакансиями приводит к образованию тетрагональной фазы, в которой кислородные вакансии локализованы преимущественно в одной из четырёх неэквивалентных позиций аниона $\mathrm{O}^{2}$. При этом параллельно с увеличением показателя нестехиометрии $\delta$ происходит снижение энтальпии отщепления $\mathrm{O}_{2}$ и изменение количества подвижного кислорода. Показано, что метод синхронного термического анализа (параллельная запись сигналов ТГ, ДСК с масс-спектральной фиксацией состава газовой фазы) является эффективной экспресс методикой оценки свойств подвижного кислорода в динамических условиях при изменении парциального давления кислорода. Наблюдаемые различия в свойствах мобильного кислорода и каталитической активности в реакции глубокого окисления метана для упорядоченной и разупорядоченной структур перовскита могут быть объяснены изменением доли анионов $\mathrm{O}^{2-}$ с различным локальным окружением, содержащим от 0 до 4 катионов гадолиния.

\section{Благодарности}

Исследование выполнено при финансовой поддержке Российского фонда фундаментальных исследований, Правительства Красноярского края, Красноярского краевого фонда поддержки научной и научно-технической деятельности (проект 16-43-240505 «р_а»), а также частично в рамках проекта фундаментальных исследований СО РАН V.45.3.3. 


\section{Список литературы}

1. Pena M.A., Fierro J.L.G. Chemical Structures and Performance of Perovskite Oxides. Chem. Rev. 2001. Vol. 101, P. 1981-2018.

2. Falcon H., Martinez-Lope M.J., Alonso J.A., Fierro J.L.G. Defect $\mathrm{LaCuO}_{3-\delta}(\delta=0.05-0.45)$ perovskites: Bulk and surface structures and their relevance in $\mathrm{CO}$ oxidation. Appl. Catal. B: Environ. 2000. Vol. 26, P. 131-142.

3. Jiang A., Peng Y., Geo K.W., Zhou P.Y., et al. The catalytic oxidation of CO on superconductor $\mathrm{Ba}_{2} \mathrm{YCu}_{3} \mathrm{O}_{7-\delta}$. Catal. Lett. 1989. Vol. 3, P. 235-245

4. Tejuca L.G., Fierro J.L.G., Tascon J.M.D. Structure and reactivity of perovskite-type oxides. Adv. Catal. 1989. Vol. 36, P.237-328.

5. Suntivich J., Gasteiger H.A., Yabuuchi N., Nakanishi H. et al. Design principles for oxygenreduction activity on perovskite oxide catalysts for fuel cells and metal-air batteries. Nature Chemistry. 2011. Vol. 3, P.546-550.

6. Yakovleva I.S., Isupova L.A., Rogov V.A. Oxygen species and their reactivity in the mechanochemically prepared substituted perovskites $\mathrm{La}_{1-\mathrm{x}} \mathrm{Sr}_{\mathrm{x}} \mathrm{CoO}_{3-\mathrm{y}}(\mathrm{x}=0-1)$. Kinetics and Catalysis. 2009. Vol. 50, No 2, P. 275-283.

7. Vereshchagin S.N., Solovyov L.A., Rabchevskii E.V., Dudnikov V.A., Ovchinnikov S.G., Anshits A.G.. Methane oxidation over A-site ordered and disordered $\mathrm{Sr}_{0.8} \mathrm{Gd}_{0.2} \mathrm{CoO}_{3-\delta}$ perovskites. Chem. Commun. 2014. Vol. 50, P. 6112-6115.

8. Vereshchagin S.N., Solov'ev L.A., Rabchevskii E.V., et al. New method for regulating the activity of $\mathrm{ABO}_{3}$ perovskite catalysts. Kinetics and Catalysis. 2015. Vol. 56, No 5, P. 640-645. DOI: 10.1134/S0023158415040199.

9. Shiiba H., Bishop C.L., Rushton M.J.D. et al. Effect of A-site cation disorder on oxygen diffusion in perovskite-type $\mathrm{Ba}_{0.5} \mathrm{Sr}_{0.5} \mathrm{Co}_{1-\mathrm{x}} \mathrm{Fe}_{\mathrm{x}} \mathrm{O}_{2.5}$. J. Mater. Chem. A. 2013. Vol. 1, P. 10345-10352.

10. Vereshchagin S., Dudnikov V., Solovyov L. DSC+TG and XRD study of order-disorder transition in nonstoichiometric Sr-Gd-cobaltate. Journal of Siberian Federal University. Chemistry. 2016. Vol. 3, No 9, P. 326-336/

11. Thermal analysis; differential thermal analysis; principles. DIN 51007:1994-06.

12. Conder. K., Pomjakushina E., Soldatov A., Mitberg E. Oxygen content determination in perovskite-type cobaltates. Materials research bulletin. 2005. Vol. 40, P. 257-263.

13. Solovyov L.A. Full-profile refinement by derivative difference minimization. Journal of Applied Crystallography. 2004. Vol. 37, P. 743-749.

14. Vereshchagin S.N., Dudnikov V.A., Shishkina N.N., Solovyov L.A. Phase transformation behavior of $\mathrm{Sr}_{0.8} \mathrm{Gd}_{0.2} \mathrm{CoO}_{3-\delta}$ perovskite in the vicinity of order-disorder transition.Thermochimica Acta. 2017. Vol. 655, P. 34-41. DOI: 10.1016/j.tca.2017.06.003

15. Politov B.V., Suntsov A.Yu., Leonidov I.A., Patrakeev M.V., Kozhevnikov V.L. Thermodynamic analysis of defect equilibration in double perovskites based on $\mathrm{PrBaCo}_{2} \mathrm{O}_{6-\delta}$ cobaltite. Journal of Solid State Chemistry. 2017. Vol. 249, P. 108-113.

16. James M., Cassidy D., Goossens D.J., Withers R.L. The phase diagram and tetragonal superstructures of the rare earth cobaltate phases $\mathrm{Ln}_{1-\mathrm{x}} \mathrm{Sr}_{\mathrm{x}} \mathrm{CoO}_{3-\delta}\left(\mathrm{Ln}=\mathrm{La}^{3+}, \mathrm{Pr}^{3+}, \mathrm{Nd}^{3+}, \mathrm{Sm}^{3+}, \mathrm{Gd}^{3+}\right.$, $\mathrm{Y}^{3+}, \mathrm{Ho}^{3+}, \mathrm{Dy}^{3+}, \mathrm{Er}^{3+}, \mathrm{Tm}^{3+}$ and $\left.\mathrm{Yb}^{3+}\right)$. Journal of Solid State Chemistry. 2004. Vol. 177, P. 18861895 . 
17. King G., Woodward P.M. Cation ordering in perovskites. J. Mater. Chem. 2010. Vol. 20, P. 5785-5796. DOI: 10.1039/b926757c.

18. Triggiani L., Muñoz-García A.B., Agostiano A., Pavone M. Promoting oxygen vacancy formation and p-type conductivity in $\mathrm{SrTiO}_{3}$ via alkali metal doping: a first principles study. Phys. Chem. Chem. Phys. 2016. Vol. 18, P. 28951-28959 .

19. Ritzmann A.M., Muñoz-García A.B., Pavone M., Keith J.A., Carter E.A. Ab Initio DFT+U Analysis of Oxygen Vacancy Formation and Migration in $\mathrm{La}_{1-\mathrm{x}} \mathrm{Sr}_{\mathrm{x}} \mathrm{FeO}_{3-\delta}(\mathrm{x}=0,0.25,0.50)$. Chem. Mater. 2013. Vol. 25, P. 3011-3019. 